Cahiers de la recherche sur les droits

Cahiers

${ }^{\text {sur les }}$ Droits fondamentaux

$10 \mid 2012$

Esclavage et travail forcé

\title{
La réforme de 2008, une réelle revalorisation du rôle du Parlement?
}

Jean-Manuel Larralde

\section{OpenEdition}

1 Journals

Édition électronique

URL : https://journals.openedition.org/crdf/5306

DOI : $10.4000 /$ crdf.5306

ISSN : 2264-1246

Éditeur

Presses universitaires de Caen

Édition imprimée

Date de publication : 1 novembre 2012

Pagination : 107-117

ISSN : 1634-8842

\section{Référence électronique}

Jean-Manuel Larralde, "La réforme de 2008, une réelle revalorisation du rôle du Parlement ? », Cahiers de la recherche sur les droits fondamentaux [En ligne], 10 | 2012, mis en ligne le 01 décembre 2013, consulté le 14 novembre 2022. URL : http://journals.openedition.org/crdf/5306 ; DOI : https://doi.org/ $10.4000 /$ crdf.5306 


\title{
La réforme de 2008 , une réelle revalorisation du rôle du Parlement?
}

\author{
Jean-Manuel LARRALDE \\ Professeur de droit public à l'Université de Caen Basse-Normandie
}

\section{Retrouver le Parlement législateur}

A. Redonner au Parlement la maîtrise de la fabrication de la loi

B. Lutter contre la perte de substance de la loi

II. Une révolution parlementaire inaboutie

A. Des innovations en «trompe-l'œil »... voire contre-productives

$B$. Une réforme ne corrigeant pas les déséquilibres de la Ve République

Le constat du déséquilibre institutionnel de la V ${ }^{e}$ République a été effectué depuis longtemps. Désireux de mettre fin aux errances des deux régimes précédents, incarnées par un Parlement trop puissant ${ }^{1}$, les promoteurs de la $\mathrm{V}^{\mathrm{e}}$ République ont opté pour un parlementarisme excessivement rationalisé. La restauration du pouvoir exécutif s'est accompagnée d'une limitation du pouvoir législatif, tant en ce qui concerne ses missions normatives que celles de contrôle. Marquant la fin de la loi «expression de la volonté générale», le texte du 4 octobre 1958 a encadré son domaine, l'a soumis à la possibilité d'un contrôle du juge constitutionnel et a fait du Gouvernement et du Premier ministre les véritables directeurs de la politique législative ${ }^{2}$. Avec l'apparition du fait majoritaire en 1962, le corset constitutionnel qui enserre le Parlement de la $\mathrm{V}^{\mathrm{e}}$ République a été renforcé par le jeu politique: l'existence d'une majorité fidèle et disciplinée, soutenant le Gouvernement en place, va rendre largement virtuelle sa responsabilité devant le Parlement qui ne sera plus qu'une chambre d'enregistrement des volontés législatives du pouvoir exécutif ${ }^{3}$. Le soutien inconditionnel du Gouvernement - et même désormais du pouvoir exécutif - à

1. Pour M. Debré, «un observateur de notre vie parlementaire aurait pu, entre les deux guerres, mais davantage encore depuis la Libération, noter cette double déviation de notre organisation politique: un Parlement accablé de textes et courant en désordre vers la multiplication des interventions de détail, mais un Gouvernement traitant sans intervention parlementaire des plus graves problèmes nationaux. Le résultat de ces deux observations conduisait à une double crise: l'impuissance de l'État du fait que l'administration était ligotée par des textes inadmissibles, la colère de la nation du fait qu'une coalition partisane placée au Gouvernement la mettait devant de graves mesures décidées sans avoir été préalablement soumises à un examen sérieux» (discours devant le Conseil d'État, 27 août 1958).

2. En les dotant de moyens d'action importants tels que la fixation de l'ordre du jour des assemblées, la possibilité de déclarer irrecevables certaines propositions ou certains amendements parlementaires, la mise en œuvre du vote bloqué ou l'engagement (devant l'Assemblée nationale) de la responsabilité du Gouvernement sur le vote d'un texte, la possibilité de donner le dernier mot à l'Assemblée nationale, l'ouverture de la discussion des projets de loi sur le texte de l'exécutif...

3. Pour le comité Vedel, «la conjugaison de ces deux effets a mis le Parlement français, et notamment l'Assemblée nationale, dans une situation de subordination excessive par rapport au pouvoir exécutif. Cette dernière est d'autant plus regrettable que la profusion normative et la complexité des textes qui marquent depuis près de deux décennies le paysage juridique français ont fait perdre à la loi la qualité qui devrait être la sienne, en même temps que le Parlement a vu se réduire une partie de son prestige et de sa confiance en lui-même» (Rapport remis au Président de la République le 15 février 1993 par le Comité consultatif pour la révision de la Constitution, JORF, n 39, 16 février 1993, p. 2544). 
la majorité parlementaire (et vice versa), a entraîné un effacement considérable de la distinction entre pouvoir exécutif et pouvoir législatif et donc une relativisation de la séparation des pouvoirs. Face à un tel Parlement malmené et soumis, chacun se souvient encore de la question posée par André Chandernagor en 1967: «un Parlement pour quoi faire?» ${ }^{4}$.

Plusieurs retouches au texte de 1958 sont bien intervenues, mais aucune n'a été de nature à bouleverser radicalement la donne ${ }^{5}$. D'une autre ampleur est apparemment la révision opérée par la loi constitutionnelle n 2008-724 du 23 juillet 2008. Modifiant pas moins de quarante-cinq articles de la Constitution, cette révision constituerait, selon les termes employés par ses promoteurs, «un ensemble cohérent, structuré et audacieux de mesures destinées à renforcer le Parlement», et à permettre de mettre fin au déséquilibre des institutions qui «ne correspond plus, dans le contexte actuel, aux exigences d'une démocratie irréprochable » ${ }^{6}$. La logique de la révision est assez simple et renvoie au «mouvement nécessaire des choses» cher à Montesquieu $^{7}$ : puisque le Parlement de la $\mathrm{V}^{\mathrm{e}}$ République est trop faible, et le pouvoir exécutif trop puissant, il convient d'encadrer et de mieux contrôler le pouvoir exécutif et, corrélativement, de renforcer profondément le Parlement ${ }^{8}$. Il s'agit donc de restaurer tout à la fois l'autorité, l'efficacité et le prestige d'un Parlement qui «vote la loi », «contrôle l'action du Gouvernement » et «évalue les politiques publiques» (art. 24 de la Constitution).

Si la revalorisation de cette institution passe évidemment surtout par de nouvelles prérogatives destinées à renforcer sa mission générale de surveillance de l'action gouvernementale ${ }^{9}$, la réactivation de sa mission législative participe également à cet objectif ${ }^{10}$. Plus précisément, la lecture de la Constitution révisée en 2008 montre que le constituant a lutté sur plusieurs fronts, en essayant tout à la fois de restaurer le prestige de la loi, de moderniser les structures parlementaires, et de limiter les immixtions gouvernementales dans la procédure législative. S'il est indéniable que la révision du 23 juillet 2008 vise à retrouver le Parlement législateur, progressivement disparu depuis les années 1960 (I), cette volonté affichée peine à se transformer en réalisations concrètes et la revalorisation opérée apparaît pour le moins inaboutie (II).

\section{Retrouver le Parlement législateur}

Le Parlement, qui incarne normalement le pouvoir législatif, s'est vu dès 1958 limité dans cette fonction, puis progressivement dépossédé par la montée en puissance du pouvoir exécutif. L'un des objectifs affichés de la loi constitutionnelle de «modernisation des institutions de la $\mathrm{V}^{\mathrm{e}}$ République» est de retrouver ce Parlement législateur, ce qui passe tout à la fois par une réappropriation de la procédure législative par les deux assemblées (A) et par une revalorisation de la norme législative (B).

\section{A. Redonner au Parlement la maîtrise de la fabrication de la loi}

Il est indéniable que l'un des axes majeurs de la réforme constitutionnelle de 2008 a pour vocation de redéfinir les rôles respectifs du pouvoir exécutif et du pouvoir législatif dans la fabrication de la loi. L'influence est ici à rechercher directement dans les travaux du comité Balladur, qui souhaitait des "assemblées ayant la maîtrise de leurs travaux $»{ }^{11}$. Les nouvelles dispositions constitutionnelles visant à faire à nouveau des deux chambres les maîtresses de la norme législative jouent sur deux leviers, en cherchant à limiter les moyens de pression ou de contrainte excessifs dont dispose le Gouvernement dans la procédure législative et en redéfinissant le calendrier parlementaire.

Concernant la volonté de redonner au Parlement une relative autonomie législative au regard de l'exécutif, le Gouvernement se voit désormais limité dans l'exercice de trois techniques qui lui permettaient jusqu'alors de fortement peser sur la procédure législative.

4. A. Chandernagor, Un Parlement pour quoi faire?, Paris, Gallimard (Idées; 122), 1967.

5. On peut ici penser aux révisions $n^{\circ} 92-554$ du 25 juin 1992 introduisant un droit de regard parlementaire sur la production des normes européennes, à la loi constitutionnelle $n^{\circ} 95-880$ du 4 août 1995 portant extension du champ d'application du référendum, instituant une session parlementaire ordinaire unique, ou encore à la loi constitutionnelle no 96-138 du 22 février 1996 instituant les lois de financement de la Sécurité sociale. Pour J.-P. Duprat, la V $\mathrm{V}^{\mathrm{e}}$ République se caractérise ainsi par l’ «insuccès du réformisme parlementaire» («L’influence de la Constitution sur la vie parlementaire", in 1958-2008, cinquantième anniversaire de la Constitution française, B. Mathieu (dir.), Paris, Dalloz, 2008, p. 263).

6. Projet de loi constitutionnelle de modernisation des institutions de la $V^{e}$ République, nº 820 , présenté par F. Fillon, Premier ministre et par R. Dati, garde des Sceaux, ministre de la Justice, enregistré à la présidence de l'Assemblée nationale le 23 avril 2008, p. 2.

7. Montesquieu, De l'esprit des lois, livre XI, chapitre 6, XXX.

8. Constat déjà effectué par le comité Balladur, qui relevait que «l'affirmation effective des droits et du rôle du Parlement est la clé de l'encadrement des attributions d'un pouvoir exécutif rénové. Elle est aussi la condition d'une plus grande confiance des citoyens dans le fonctionnement de la démocratie» (Comité de réflexion et de proposition sur la modernisation et le rééquilibrage des institutions de la $V^{e}$ République, Une Ve République plus démocratique, 29 octobre 2007 , p. 30 ).

9. Nouveaux dispositifs (souvent inspirés de techniques éprouvées en droit constitutionnel américain) tels que le contrôle de l'envoi de troupes en opérations à l'étranger (art. 35 de la Constitution), le contrôle de certaines nominations présidentielles (art. 13), le contrôle des politiques publiques (art. 24).

10. Comme l'indique J.-L. Warsmann, «le renforcement du pouvoir législatif et, en particulier, de ses fonctions premières de vote de la loi et de contrôle de l'action gouvernementale, passe d'abord par une amélioration de son fonctionnement, ce qui implique de lui permettre d'adapter ses structures, de contrôler son propre agenda et d'assurer un meilleur échange avec la société grâce à la publicité de ses travaux. Cela passe ensuite par la promotion de procédures législatives proprement dites plus efficaces et de capacités de contrôle plus fortes " (Rapport $n^{\circ}$ 10o9 sur le projet de loi constitutionnelle de modernisation des institutions de la $V^{e}$ République, enregistré à la présidence de l'Assemblée nationale le 2 juillet 2008).

11. Une Ve République plus démocratique, p. 30. 
L'innovation apparaissant comme la plus spectaculaire en la matière renvoie aux modalités de fixation de l'ordre du jour, qui, selon le nouvel article 48 de la Constitution, est désormais arrêté par la Conférence des présidents de chaque assemblée et non plus par le Gouvernement. Est également porté à un jour de séance par mois l'ordre du jour réservé à l'initiative des groupes parlementaires minoritaires et d'opposition. L'idée est ici d'instaurer une «logique de concertation et de consensus ${ }^{12}$. La réforme de 2008 met ainsi fin à une spécificité française, voulue par les constituants de 1958, qui souhaitaient éviter tout dérapage en raison du poids important des partis politiques et avaient donc refusé au Parlement, comme cela est normalement le cas dans tous les régimes démocratiques, de disposer librement de son ordre du jour, lequel reste toutefois partagé en pratique, puisque deux semaines de séance sur quatre sont réservées au Gouvernement qui peut toujours déterminer la liste des projets et propositions de loi qu'il désire voir examiner, ainsi que leur ordre. Au-delà de cette priorité gouvernementale «ordinaire», l'article 48 prévoit même une "super priorité » gouvernementale, puisqu'il indique que l'examen des projets de loi de finances, des projets de lois de financement de la Sécurité sociale, des projets de prorogation de l'envoi de troupes en opérations extérieures au-delà de quatre mois, des projets relatifs aux États de crise est inscrit «par priorité » à la demande du Gouvernement. L'examen de ces différents textes n'est donc pas imputé sur le quota de deux semaines réservé au Gouvernement.

Dans un souci de modifier la «machinerie législative», le constituant a également fortement revalorisé les commissions parlementaires (dont le nombre est désormais porté à huit dans chacune des deux assemblées), qui avaient vu leur rôle canalisé en 1958, en raison de leur influence jugée néfaste sous la République précédente $^{13}$. Avant la révision de 2008, s'agissant des projets de loi, la discussion en séance publique portait, devant la première assemblée saisie, sur le texte présenté par le Gouvernement (art. 42 de la Constitution). Confortable pour le Gouvernement, cette mesure lui permettait, s'il ne souhaitait pas que son texte soit trop transformé lors de la discussion parlementaire, d'écarter les amendements qui ne lui convenaient pas en utilisant pour cela les outils constitutionnels à sa disposition (tels que l'art. 44 al. 3 de la Constitution). Désormais, l'examen des projets de loi porte sur la version de la commission (s'alignant donc sur ce qui était déjà prévu pour les propositions de loi). On peut penser que cette innovation contraindra le Gouvernement à participer davantage et mieux aux séances des commissions pour y défendre son texte ${ }^{14}$. Lors de la séance plénière de l'Assemblée, il lui sera nécessaire d'argumenter et de défendre sa position s'il souhaite convaincre les parlementaires de la supériorité de ses dispositions sur les modifications introduites par la commission ${ }^{15}$. Ce nouveau processus pourrait également améliorer le déroulement de la procédure, en purgeant les séances publiques de la discussion d'amendements techniques et rédactionnels qui n'aboutissent qu'à obscurcir et ralentir les débats: les discussions purement techniques ayant eu lieu en amont, seuls les points essentiels seraient évoqués lors de la séance publique, qui retrouverait alors un caractère beaucoup plus politique ${ }^{16}$. Pour certains commentateurs, la modification de l'article 42 ainsi opérée est à la fois «innovante» et «fondamentale», car cette nouvelle disposition traduirait "l'abandon d'un parlementarisme rationalisé excessif et amplifié par le fait majoritaire ${ }^{17}$. Ce volet de la réforme nécessite indéniablement une présence accrue des parlementaires, tant en commission qu'en séance plénière. À ce titre, l'on peut regretter que la révision de 2008 fasse l'impasse sur l'une des réformes structurelles du comité Balladur, qui avait proposé l'abandon de tout cumul entre un mandat national et l'exercice d'une fonction exécutive locale ${ }^{18}$.

L'article 49 alinéa 3 de la Constitution, «instrument emblématique du parlementarisme rationalisé ${ }^{19}$, a également été affecté par les modifications constitutionnelles. Destiné dans l'esprit du constituant à être utilisé avec parcimonie, pour des textes particulièrement importants pour la réalisation du programme du Gouvernement, il a été progressivement détourné de son usage premier, pour

12. Projet de loi constitutionnelle..., p. 6.

13. Pour M. Debré, les commissions permanentes ont eu tendance, sous la IV ${ }^{e}$ République, «à déborder de leur mission, à se substituer aux assemblées et à empiéter sur les prérogatives des ministres" (Comité national chargé de la publication des travaux préparatoires des institutions de la $\mathrm{V}^{\mathrm{e}}$ République, Documents pour servir à l'histoire de l'élaboration de la Constitution du 4 octobre 1958, vol. I, Paris, La documentation française, 1987, p. 325).

14. Dans sa décision $n^{\circ}$ 2009-579 DC du 9 avril 2009 (loi organique relative à l'application des articles $34-1$, 39 et 44 de la Constitution), le Conseil constitutionnel a bien indiqué que les nouvelles dispositions constitutionnelles «impliquent que le Gouvernement puisse participer aux travaux des commissions consacrés à l'examen des projets et des propositions de loi ainsi que des amendements dont ceux-ci font l'objet et assister aux votes destinés à arrêter le texte sur lequel portera la discussion en séance» (cons. 36). F. Fillon a également rappelé aux ministres de participer de manière active aux travaux en commission, afin de faire utilement valoir le point de vue du Gouvernement sur le texte défendu (Circulaire du 15 avril 2009 relative à la mise en œuvre de la révision constitutionnelle, JORF, nº 0089,16 avril 2009, p. 6546).

15. Voir T. Cartier-Molin, «La portée du nouveau rôle législatif des commissions parlementaires (commentaire des deux premiers alinéas de l’article 42 de la Constitution)», Revue du droit public, no 5 , 2010, p. 1399.

16. J.-L. Warsmann, «Le nouveau rôle du Parlement après la révision », L'ENA hors les murs, n 385, octobre 2008 , p. 29.

17. P. Jan, «Les débats des projets à partir du texte de la commission: disposition innovante... sous conditions ou risque d'un nouveau déséquilibre?», Les petites affiches, $\mathrm{n}^{\circ} 254,2008, \mathrm{p} .68$. Pour cet auteur, l'article 42 constituerait même «la pierre angulaire d'un déséquilibre retrouvé entre le législatif et l'exécutif et d'une revanche du premier sur le second» (ibid., p. 69).

18. Une Ve République plus démocratique, proposition $\mathrm{n}^{\circ} 68, \mathrm{p} .65$.

19. Projet de loi constitutionnelle..., p. 7 . 
devenir une arme de contrainte contre une Assemblée nationale montrant des velléités d'indépendance vis-àvis du Gouvernement ${ }^{20}$ : utilisé quatre-vingt-deux fois depuis 1958 (une motion de censure ayant été déposée en réponse à son application à quarante-huit reprises), il a permis l'adoption de quarante-huit textes (aucune utilisation au cours de l'actuelle législature, seulement trois sous la précédente). Dans sa version entrée en vigueur le $1^{\text {er }}$ mars 2009, ce dispositif n'est plus utilisable que pour le vote d'un projet de loi de finances ou de financement de la Sécurité sociale et, pour le surplus, pour un autre texte par session. Certains commentateurs n'ont pas manqué de critiquer cette limitation, en soulignant que l'article 49 alinéa 3 de la Constitution constitue « un élément essentiel pour la stabilité de la $V^{\mathrm{e}}$ République» et que sa limitation serait notamment de nature à rendre la situation d'un Gouvernement minoritaire intenable ${ }^{21}$.

La réforme cherche également à peser sur la gestion du temps dans l'élaboration de la loi, en imposant un calendrier plus respectueux des contraintes parlementaires. Déposés parfois à la hâte, et fréquemment trop peu de temps avant leur examen, de nombreux projets de lois ne permettent pas aux rapporteurs des commissions saisies au fond de travailler de manière approfondie et rigoureuse. Les amendements déposés en commission apparaissent alors insuffisamment préparés. L'idée est ici indéniablement d'obliger le Gouvernement «à plus de tempérance normative ${ }^{22}$. Dans cette optique, l'article 42 alinéa 3 de la Constitution prévoit désormais que:

[l]a discussion en séance, en première lecture, d'un projet ou d'une proposition de loi ne peut intervenir, devant la première assemblée saisie, qu'à l'expiration d'un délai de six semaines après son dépôt. Elle ne peut intervenir, devant la seconde assemblée saisie, qu'à l'expiration d'un délai de quatre semaines à compter de sa transmission.
Ces délais renvoient directement à la volonté de revalorisation de la commission saisie au fond, qui devrait être désormais assurée de bénéficier de délais suffisants. La volonté est ici claire : éviter le vote de lois bâclées en raison d'un manque d'examen lors du passage en commission. Corrélativement, la phase de l'examen en séance publique devrait s'en trouver revalorisée ${ }^{23}$.

Cette restauration d'un Parlement législateur ${ }^{24}$ ne passe pas seulement pas une refonte des méthodes et calendriers parlementaires. Elle renvoie également à la volonté de restaurer la norme législative.

\section{B. Lutter contre la perte de substance de la loi}

Aux yeux du constituant de 2008 , le renforcement du Parlement législateur nécessite de redonner un certain «lustre» à la loi. Votée trop vite, dans de trop nombreux domaines, celle-ci est même aujourd'hui accusée de ne plus systématiquement posséder un contenu normatif ${ }^{25}$. La loi française contemporaine souffrirait donc d'une perte importante de substance. Depuis 2004, le Conseil constitutionnel démontre ainsi une volonté de lutter contre ce mouvement de dévalorisation de la loi $^{26}$. Dans sa décision $\mathrm{n}^{\circ} 2004-500 \mathrm{DC}$ du 29 juillet 2004 (loi organique relative à l'autonomie financière des collectivités territoriales), il a ainsi rappelé qu'il résulte de l'article 6 de la Déclaration des Droits de l'homme et du citoyen de 1789 que «la loi a pour vocation d'énoncer des règles et doit par suite être revêtue d'une portée normative» (cons. 12). Le discours prononcé par Pierre Mazeaud, lors de la présentation des vœux 2005 du Conseil constitutionnel au chef de l'État ${ }^{27}$, puis la décision no 2005-512 DC du 21 avril 2005 qui déclare, pour la première fois, contraire à la Constitution certaines dispositions d'une loi car «dépourvues

20. L'article 49 alinéa 3 a ainsi permis de resserrer une majorité faible ou divisée (1958-1962; 1967-1968; 1976-1981; 1988-1993) ou de contraindre une majorité forte à accepter des dispositions pouvant la choquer (amnistie des généraux d'Alger sous la présidence de F. Mitterrand en 1982, par exemple).

21. Voir notamment M. Lascombe, «Le Gouvernement et la révision constitutionnelle: un oublié très présent ", La semaine juridique, éd. G, n $31-35$, 2008, I, 173, p. 36; J. Giquel, «Sauvegarder l’article 49, alinéa 3!», Les petites affiches, nº 254, 2008, p. 91 sq.

22. Pour reprendre les termes employés par le comité Balladur (Une Ve République plus démocratique, p. 49).

23. Cet argument ayant déjà été mis en avant par le comité Balladur, qui soutenait que "pour améliorer le travail en séance publique, il convient de lui donner un caractère plus politique que technique, ce qui implique que la discussion s'engage non plus sur le texte du Gouvernement mais sur celui de la commission. Il ne faut pas s'y tromper, c'est là une transformation fondamentale du travail parlementaire et gouvernemental. Cela suppose aussi que le nombre des commissions permanentes soit accru, que celles-ci puissent examiner les lois les plus simples ou les plus techniques, les séances publiques étant alors réservées au seul vote solennel du texte, et que le Gouvernement se plie à une exigence nouvelle, essentielle au bon fonctionnement des institutions: donner plus de temps aux commissions pour travailler, et participer lui-même à ce travail» (Une Ve République plus démocratique, p. 45).

24. Qui semble être un succès d'un point de vue au moins statistique, car sur les cinquante et une lois adoptées par le Parlement depuis le $1^{\text {er }}$ janvier 2010 (chiffre au 30 novembre 2010, hors lois de ratifications de conventions internationales), vingt-neuf sont des projets, vingt-deux sont des propositions. Mais le contenu respectif de ces lois tempère tout enthousiasme excessif. Sans presque caricaturer les choses, on pourrait dire qu'aux parlementaires revient de proposer des lois rendant obligatoires les détecteurs de fumée dans tous les lieux d'habitation (loi $\mathrm{n}^{\mathrm{o}} 2010-238$ du 9 mars 2010) ou tendant à suspendre la commercialisation de biberons produits à base de bisphénol A (loi n $2010-729$ du 30 juin 2010$)$ et au Premier ministre les lois relatives à la protection des sources journalistiques (loi no 2010-1 du 4 janvier 2010) ou à la réforme des retraites (loi $\mathrm{n}^{\circ}$ 2010-1330 du 9 novembre 2010)...

25. «Le caractère non normatif de certaines lois, soit que la loi ne contient pas de dispositions normatives soit en ce qu'elle n'est pas à l'origine des dispositions normatives qu'elle contient, participe manifestement de la crise que subit la règle impérative comme mode de régulation sociale» (B. Mathieu, La loi, Paris, Dalloz (Connaissance du droit), 1996, p. 98).

26. Dans le même sens, J.-L. Debré, alors président de l'Assemblée nationale, avait déposé le 5 octobre 2004 une proposition de loi constitutionnelle $\left(n^{\circ} 1832\right)$ qui visait à rejeter ce qui est du ressort du règlement dans les textes de loi, afin d'alléger leur contenu ainsi que les travaux parlementaires.

27. Les Cahiers du Conseil constitutionnel, $\mathrm{n}^{\circ} 18,2005$, p. 3 sq. 
de toute portée normative» (et reconnaît préventivement le caractère réglementaire d'autres dispositions de cette même loi), ont confirmé la volonté du juge constitutionnel de lutter contre la perte de normativité de la loi. On sait par ailleurs que les présidents des deux assemblées ont décidé, le 6 avril 2010, de mettre en place un groupe de travail chargé d'améliorer la qualité de la loi et les conditions de son élaboration par les parlementaires ${ }^{28}$. Face à ces critiques convergentes, la révision du 23 juillet 2008 essaie de lutter sur trois fronts: renforcer le domaine de la loi, lui redonner un caractère normatif plus affirmé et enfin rationaliser sa production.

Afin de renforcer la place de la norme législative, la loi constitutionnelle $n^{\circ}$ 2008-724 a tout d'abord opéré un renforcement par petites touches de l'article $34^{29}$ qui délimite depuis 1958 le domaine de la loi ${ }^{30}$. Désormais, il lui revient aussi de déterminer les règles relatives à «la liberté, du pluralisme et de l'indépendance des médias », au « régime électoral des instances représentant les Français établis hors de France» et aux «conditions d'exercice des mandats électoraux et des fonctions électives des membres des assemblées locales».

Il revient également désormais à la loi de fixer les principes fondamentaux de la «recherche». Par ailleurs, le nouveau libellé de l'article $1^{\text {er }}$ de la Constitution fait de la loi la norme chargée de favoriser «l'égal accès des femmes et des hommes aux responsabilités professionnelles et sociales », de la même manière qu'elle devait déjà favoriser depuis 1999 un égal accès aux mandats électoraux et fonctions électives.

Au-delà de cette extension limitée du domaine législatif, la révision constitutionnelle de 2008 cherche à améliorer la qualité de la loi en renforçant son caractère normatif. Ceci a abouti à mettre en place deux techniques, qui permettent au Parlement d'exprimer des positions, sans pour autant utiliser la voie de la loi ordinaire.

Avec l'article 34 modifié de la Constitution apparaissent les lois de programmation, qui élargissent le champ d'application des anciennes lois de programme. Ces dernières permettaient d'associer le Parlement à la définition des objectifs gouvernementaux, mais uniquement dans le domaine économique et social. Il est désormais possible au Parlement d'adopter des lois de programmation qui « déterminent les objectifs de l'État». Le caractère de catalogue d'objectifs (non normatifs, ou d'une normativité incertaine) qui caractérisait les lois de programme se retrouve désormais pour les lois de programmation qui s'appliquent à tous les domaines de l'action gouvernementale. Par ailleurs, le Parlement est désormais habilité à adopter, par une loi de programmation, des orientations pluriannuelles des finances publiques et ce, dans l'objectif d'équilibre des comptes des administrations publiques (avant-dernier alinéa de l'art. 34 de la Constitution). L'intention du constituant en la matière est probablement louable, mais on peut s'interroger sur la nouvelle portée des lois de "programmation» au regard des défuntes lois de «programme». Comme l'indique justement Xavier Vandendriessche, il était possible de retenir une conception très large du domaine "économique et social» sur lequel portaient ces lois de programme, et donc «admettre sans peine que toute politique publique peut se rattacher à la vie économique et sociale $»^{31}$.

Par ailleurs, avec le nouvel article 34-1 de la Constitution est réintroduite pour le Parlement la possibilité de voter des résolutions. Utilisées de manière excessive sous les deux Républiques précédentes, ces résolutions ont été cantonnées à partir de 1958 à des domaines techniques et très limités ${ }^{32}$. Elles font aujourd'hui leur réapparition de manière plus générale. Comme l'a précisé la commission des lois de l'Assemblée nationale en première lecture, ces résolutions, "n'ayant pas de valeur contraignante, permettront de marquer l'expression d'un souhait ou d'une préoccupation; déchargée de cette fonction tribunitienne, la loi pourra retrouver son caractère normatif ${ }^{33}$. L'idée est ici de recentrer l'action législative du Parlement autour de l'adoption des seuls textes créant des droits ou des obligations, la technique des résolutions évitant désormais d'utiliser la loi pour des prises de position politiques ou l'adoption de ce que l'on a pu appeler des «lois mémorielles $»^{34}$. Par le vote de résolutions le Parlement pourra

28. Pour le président du Sénat, Gérard Larcher, qui a déploré le «volume» et l' «usage abusif de la procédure accélérée» et le «délai entre le dépôt d'un projet et son examen par la commission», «il faut améliorer l'efficacité et l'intelligibilité de la loi» (http://www.publicsenat.fr/lcp/politique/ gerard-larcher-bernard-accoyer-se-penchent-qualite-loi-9564).

29. Article qui avait déjà été complété par les lois constitutionnelles no 96-138 du 22 janvier 1996, qui crée la catégorie des lois de financement de la Sécurité sociale et $\mathrm{n}^{\mathrm{o}}$ 2005-205 du $1^{\mathrm{er}}$ mars 2005, qui ajoute la «préservation de l'environnement» aux domaines dont la loi détermine les principes fondamentaux.

30. Même si l'on sait que cette limitation est devenue partiellement inopérante à partir de 1982, date à partir de laquelle le Conseil constitutionnel a estimé que les articles 34 et 37 de la Constitution ne font pas obstacle à ce que le Parlement puisse légiférer dans un domaine relevant en principe du pouvoir réglementaire (déc. n 82-143 DC du 30 juillet 1982, «Blocage des prix»).

31. X. Vandendriessche, «Une revalorisation parlementaire à principes constitutionnels constants», La semaine juridique, éd. G, nº 31,30 juillet $2008, \mathrm{I}, 174, \S 26$.

32. Modifier le règlement des assemblées, créer une commission d'enquête, suspendre les poursuites ou la détention d'un membre d'une assemblée, mettre en accusation le président de la République devant la Haute Cour, définir une position sur des textes de l'Union européenne soumis au Parlement dans le cadre de l'article 88-4 de la Constitution.

33. J.-L. Warsmann, Rapport $n^{\circ} 892$ fait au nom de la commission des lois constitutionnelles, de la législation et de l'administration générale de la République sur le projet de loi constitutionnelle $\left(n^{\circ} 820\right)$ de modernisation des institutions de la Ve République, enregistré à la présidence de l'Assemblée nationale le 15 mai 2008, p. 251.

34. Telles que la loi $n^{\circ} 2001-70$ du 29 janvier 2001 relative à la reconnaissance du génocide arménien de 1915, la loi nº 2001-434 du 21 mai 2001 tendant à la reconnaissance de la traite et de l'esclavage en tant que crime contre l'humanité, ou encore la loi no 2005 -158 du 23 février 2005 portant reconnaissance de la Nation et contribution nationale en faveur des Français rapatriés, qui prévoyait que les manuels scolaires devaient rendre compte de la présence française outre-mer et de son « rôle positif». 
adopter des textes qui n'auront pas de force obligatoire et ne lieront pas le Gouvernement. Plus de dix-huit mois après la mise en place de cette nouvelle procédure, force est toutefois de constater que le Parlement s'est encore relativement peu saisi de la technique des résolutions ${ }^{35}$.

On peut, enfin, relever la volonté de rationaliser la production législative par la mise en place des études d'impact ${ }^{36}$. L'idée est ici d'envisager a priori les objectifs des effets de la loi et de s'assurer que toutes les conséquences de ce texte, qu'elles soient juridiques, économiques, sociales ou budgétaires ont bien été prises en compte ${ }^{37}$. Aux termes de la loi organique, cette étude, prise en charge par le ministre responsable du projet de loi concerné, doit désormais définir «les objectifs poursuivis par le projet», recenser «les options possibles en dehors de l'intervention de règles de droit nouvelles» et exposer «les motifs du recours à une nouvelle législation ». On peut espérer que cette nouvelle technique permettra de contribuer à la lutte contre les lois de circonstance, déposées dans la précipitation par le Premier ministre et qui aboutissent à des doublons inutiles ${ }^{38} \mathrm{ou}$ ne font qu'ajouter un nouveau texte dans une matière déjà particulièrement bien fournie en normes législatives ${ }^{39} .$.

Les innovations opérées par la loi constitutionnelle $\mathrm{n}^{\circ}$ 2008-724 sont indéniables et certaines sont même particulièrement originales (on peut ici notamment penser à l'instauration des études d'impact). Mais leurs conséquences concrètes sur la revalorisation de l'institution parlementaire risquent d'être relativement limitées, car la réforme mise en place est largement inaboutie.

\section{Une rénovation parlementaire inaboutie}

Si l'on en croit le Gouvernement,

[1]a session 2009-2010, entièrement régie par les nouvelles règles de procédure parlementaire issues de la révision constitutionnelle du 23 juillet 2008, marque une importante revalorisation du Parlement. Députés et sénateurs, de la majorité comme de l'opposition, ont pleinement utilisé les droits nouveaux qui leur ont été reconnus ${ }^{40}$.

On peut toutefois opter pour une autre analyse et penser, au contraire, que les résultats de la révision du 23 juillet 2008 restent encore fort limités. En effet, les différentes innovations opérées par la loi constitutionnelle sont tout à la fois en "trompe-l'œil», trop partielles, et même potentiellement contre-productives pour certaines d'entre elles (A). Elles semblent, en outre, impuissantes à dépasser un obstacle majeur, qui est celui de la nature même du régime: les modifications opérées ne remettent en effet pas en cause les grands principes qui sous-tendent aujourd'hui le fonctionnement de la $\mathrm{V}^{\mathrm{e}}$ République (B).

\section{A. Des innovations en «trompe-l'œil »... voire contre-productives}

Comme l'indique Olivier Dord, «le succès de la révision [...] est subordonné à la modération dont le Gouvernement saura faire preuve dans l'utilisation des différents privilèges qu'il conserve $[. ..] »^{41}$. Si la remarque de l'auteur renvoie uniquement aux nouvelles modalités de partage de l'ordre du jour instaurées par l'article 48 alinéa $1^{\text {er }}$ de la Constitution, ce constat est en réalité relativement inquiétant, car il peut s'appliquer à l'essentiel des réformes de 2008. Une lecture attentive de la Constitution révisée, à laquelle s'ajoutent déjà quelques éléments de mise en œuvre, démontre en effet que la volonté de rationaliser le Parlement, présente dans la loi constitutionnelle du 23 juillet 2008, souffre de maux importants. Ceux-ci résident tout à la fois dans le caractère factice de plusieurs innovations et dans la possibilité toujours reconnue au Gouvernement de mobiliser différents outils constitutionnels qui restent à sa disposition. Ces deux risques immédiatement identifiables pourraient même être aggravés par l'émergence rapide de pratiques contraires à l'esprit de la réforme.

35. Au 30 novembre 2010 ont été déposées vingt-quatre propositions de résolutions, et seulement trois d'entre elles ont fait l'objet d'une adoption : deux par l'Assemblée nationale (résolution du 25 mars 2010 incitant le Gouvernement français à remplir les obligations que lui donnent les recommandations de la commission d'enquête tchadienne concernant les événements du 28 janvier au 8 février 2008 afin de connaître la situation de M. Ibni Oumar Mahamat Saleh disparu à N’Djamena, capitale du Tchad, le 3 février 2008; résolution du 11 mai 2010 sur l'attachement au respect des valeurs républicaines face au développement de pratiques radicales qui y portent atteinte) et une par le Sénat (résolution du 28 juin 2010 relative à la mise en œuvre de la contribution économique territoriale).

36. Art. 8 de la loi organique $\mathrm{n}^{\circ} 2009-403$ du 15 avril 2009.

37. Comme le souligne le Rapport public du Conseil d'État de 2006, cette évolution française s'inscrit dans un mouvement beaucoup plus large que connaissent les démocraties, de revalorisation de la qualité de la loi; relèvent d'une même logique les «Regulatory Impact Assessments» que le «Cabinet office» exige depuis 1998 au Royaume-Uni, ainsi que la loi sur les responsabilités extrabudgétaires («Unfunded Mandates Reform Act») adoptée aux États-Unis, ou la réforme de ses méthodes de travail gouvernementales adoptée par l'Espagne en novembre 1997 (Conseil d'État, Rapport public 2006. Sécurité juridique et complexité du droit, Paris, La documentation française (Études et documents du Conseil d'État), 2006).

38. On peut penser ici par exemple à la délicate articulation de la loi no 2007-297 du 5 mars 2007 relative à la prévention de la délinquance avec la loi n 2006-396 du 31 mars 2006 pour l'égalité des chances (tout spécialement sur la question de l'«accompagnement parental»).

39. Ainsi, concernant la seule question des chiens dangereux, une loi a été adoptée le 6 janvier 1999 ( ${ }^{\circ}$ 99-5), de nouvelles dispositions ont été adoptées le 5 mars 2007 au sein de la loi relative à la prévention de la délinquance (n²007-297) et une nouvelle loi a été adoptée le 20 juin 2008 (n 2008-582). D’une manière plus générale, entre 2002 et 2008 ont été adoptées pas moins de dix-sept lois relatives à la sécurité et à la lutte contre la délinquance...

40. «Le bilan de la session parlementaire», extrait du Conseil des ministres du 21 juillet 2010.

41. O. Dord, "Vers un rééquilibrage des pouvoirs publics en faveur du Parlement ", Revue française de droit constitutionnel, $\mathrm{n}^{\circ}$ 77, 2009, p. 101-102. Dans le même sens, voir A. Levade, «Les nouveaux équilibres de la Ve République», Revue française de droit constitutionnel, n 82, 2010 , p. 244. 
Ainsi, la limitation de l'utilisation de l'article 49 alinéa 3 de la Constitution ne concernera que des situations marginales. En effet, seuls des Premiers ministres particulièrement malmenés par la majorité parlementaire ont été contraints d'utiliser de manière massive cette procédure ${ }^{42}$. Ni Dominique de Villepin, ni François Fillon (que ce soit avant ou après mars 2009) auraient vraiment été gênés dans leur action gouvernementale par l'encadrement de cette procédure d'engagement de responsabilité gouvernementale sur le vote d'un texte ${ }^{43}$. Et comme le mentionnait déjà le comité Balladur, «il apparaît que parmi les textes le plus souvent adoptés grâce à l'article 49, alinéa 3, figurent au premier rang les lois de finances et les lois portant sur la matière sociale ${ }^{44}$. Le rétrécissement du champ d'application matériel de cette disposition semble donc largement inutile et d'une portée pratique très limitée ${ }^{45}$.

Le partage - semble-t-il égalitaire - entre l'ordre du jour gouvernemental et parlementaire renvoie également à des faux-semblants, car cette apparente répartition paritaire ne s'applique pas à des propositions particulièrement importantes ${ }^{46}$. Ce nouvel article 48 pourrait poser des difficultés concrètes à des gouvernements atteints de "frénésie législative» et qui n'auraient déjà pas le temps de faire adopter tous leurs projets. Or, ce problème n'en est pas vraiment un, car, hormis le recours à des sessions extraordinaires (deux chaque année depuis 2007), l'article 48 pourra facilement être détourné dans la pratique. En premier lieu, le Gouvernement pouvant faire inscrire prioritairement à l'ordre du jour des textes transmis depuis plus de six semaines, il lui suffira de laisser le temps passer pour les convertir en projets prioritaires. D'autre part, rien n'interdit au Gouvernement de faire pression sur sa majorité parlementaire pour faire inscrire dans l'ordre du jour normalement réservé aux parlementaires des projets de loi qu'il souhaite voir adoptés au plus vite. Cette possibilité a d'ailleurs vite été comprise et mise en œuvre et elle a ainsi été utilisée au Sénat en mai 2009 pour l'adoption de la loi no 2009-879 du 21 juillet 2009 portant réforme de l'hôpital et relative aux patients, à la santé et aux territoires et en septembre 2009 pour l'adoption du projet de loi HADOPI (loi n 2009-669 du 12 juin 2009 favorisant la diffusion et la protection de la création sur Internet).
De même, la mise en place d'un nouveau calendrier parlementaire par l'article 42 alinéa 3 laisse largement de côté l'essentiel. Il est certes louable que la discussion en séance ne débute pas avant l'expiration d'un délai de six semaines suivant le dépôt du texte et que la seconde assemblée ne débute pas l'examen du texte moins de quatre semaines après sa transmission. Mais le problème essentiel de la contraction du calendrier parlementaire ne réside pas vraiment ici. On sait, en effet, que les gouvernements successifs ont abusé de la procédure prévue par l'article $45 \mathrm{de}$ la Constitution, qui permet au Premier ministre d'invoquer l'urgence après une seule lecture par chaque assemblée. D'exceptionnelle, celle-ci est devenue courante dans la procédure parlementaire. Et elle a abouti à priver de navette nombre de textes, pour lesquels on chercherait en vain la nécessité de cette accélération! La deuxième lecture n'est plus le droit commun et ce raccourcissement de la procédure législative constitue l'un des éléments d'explication de la mauvaise qualité de certaines lois, qui, adoptées après une seule lecture, n'ont pas pu subir le travail d'amélioration et de perfectionnement du texte que permettent les lectures successives ${ }^{47}$. La déclaration d'urgence constitue surtout un moyen pour le Gouvernement d'organiser l'encombrement chronique de l'ordre du jour parlementaire (et l'on constate fort logiquement que l'urgence est davantage utilisée en fin de législature qu'en début...).

Cette dérive semble bien avoir été prise en compte par le constituant de 2008 puisque l'on ne trouve plus trace de l' «urgence» dans la Constitution. Celle-ci est remplacée par la procédure dite "accélérée», qui prévoit toujours, sur demande du Premier ministre, de limiter l'examen du texte à une seule lecture par les deux assemblées. Un verrou a bien été ajouté, car le nouvel article 45 alinéa 2 de la Constitution prévoit que la Conférence des présidents peut s'opposer à cette procédure accélérée. Ce veto apparaît toutefois dérisoire en pratique, car la composition de cette institution ${ }^{48}$ ne permet pas de penser qu'elle pourra s'opposer à la volonté du Premier ministre, comme le montre l'adoption de la loi portant dissimulation du visage dans le domaine public (loi no 2010-1192 du 11 octobre 2010), qui a entraîné des remous même au sein des parlementaires de la majorité, mais sans que la procédure accélérée soit pour autant

42. Tels Michel Rocard avec vingt-huit utilisations entre 1988 et 1991; ou encore Édith Cresson avec huit utilisations en 1991-1992.

43. Dominique de Villepin a, en effet, utilisé cette procédure à une seule reprise entre 2005 et 2007 . Quant à François Fillon, il ne l'a jamais mise en œuvre depuis 2007. L'article 49 alinéa 3 a été utilisé seulement à trois reprises entre 2002 et 2007, et à chaque fois pour mettre fin à des pratiques d'obstruction. Voir P. Avril, «Les conditions d'une revalorisation du rôle du Parlement», Les cahiers français, n 332, mai-juin 2006 , p. 53.

44. Une Vépublique plus démocratique, p. 34.

45. Certaines propositions en la matière ont été plus radicales et auraient pu changer réellement le rapport des forces. Ainsi, dans la proposition de loi constitutionnelle $n^{\circ} 386$ tendant à réviser la Constitution du 4 octobre 1958 afin de rééquilibrer les institutions en renforçant les pouvoirs du Parlement, présentée par J.-P. Bel et des membres du groupe socialiste et apparentés du 12 juillet 2007, ces sénateurs souhaitaient «desserrer le carcan du contrôle gouvernemental sur le travail parlementaire en supprimant l'article 49, alinéa 3 ainsi que le vote bloqué, en limitant le nombre de déclarations d'urgence à 10 par an, en restreignant le domaine des ordonnances».

46. Voir n. 24.

47. Pour J.-E. Gicquel, cette procédure d'urgence a fortement « déréglé le déroulement de la discussion parlementaire et porté atteinte au fonctionnement correct du bicamérisme» ( «L'article 19. L’indispensable révision d’utilisation de la déclaration d’urgence», Les petites affiches, n 97, 2008, p. 61).

48. Prévue par le règlement des assemblées, la Conférence des présidents comprend les vice-présidents, les présidents des groupes, les présidents des commissions permanentes et d'autres commissions précisées par le règlement de chaque chambre (voir les art. 47 al. $1^{\text {er }}$ du Règlement de l'Assemblée nationale (RAN) et 29 al. $1^{\text {er }}$ du Règlement du Sénat (RS)). 
écartée ${ }^{49}$. Et l'on peut également douter de la nécessité d'accélérer la procédure législative pour adopter une loi relative à l'équipement numérique des établissements de spectacles cinématographiques (loi no 2010-1149 du 30 septembre 2010). De même, si l'on peut comprendre l'urgence sociale en la matière, l'utilisation de la procédure accélérée pour l'adoption de la très importante loi $\mathrm{n}^{\circ}$ 2010-1330 du 9 novembre 2010 portant réforme des retraites est de nature à affaiblir le débat démocratique et à accroître le risque d'adopter une loi imparfaite qui ne sera pas passée par toutes les étapes de la fabrication législative. D'ailleurs, dans son rapport annuel de contrôle de l'application des lois de décembre 2009, le Sénat indique bien que le recours à

[...] la déclaration d'urgence ou, depuis mars 2009, à la procédure accélérée [...] tend à devenir la norme pour la plupart des projets de loi, et même pour des propositions de loi, dans le cadre des nouvelles dispositions régissant l'ordre du jour instaurées par la réforme constitutionnelle du 23 juillet 2008. En effet, le Gouvernement ne dispose plus (hors loi de finances initiale et loi de financement de la sécurité sociale) que de deux semaines sur quatre dans chaque assemblée pour faire voter ses textes, sauf durant les sessions extraordinaires où le Gouvernement conserve la maîtrise de la totalité de l'ordre du jour ${ }^{50}$.

Enfin, même la réforme pourtant présentée comme la plus intéressante, et qui impose que l'examen des projets de loi porte sur la version de la commission, risque de générer des effets pervers, en renforçant encore un peu plus les liens entre l'exécutif et sa majorité parlementaire. Le Conseil constitutionnel, dans sa décision $\mathrm{n}^{\circ}$ 2009-579 du 9 avril 2009, a bien précisé que la nouvelle donne constitutionnelle exige que le Gouvernement puisse bénéficier en commission de prérogatives étendues (cons. 36). En outre, celui-ci devra davantage se battre en commission pour défendre son texte ${ }^{51}$. Ainsi, lors de la discussion du projet de loi dit «Grenelle II ( (devenu la loi n 2010-788 du 12 juillet 2010 portant engagement national pour l'environnement), on sait qu'en faisant pression sur sa majorité à l'Assemblée nationale, il a réussi à la faire revenir sur le texte voté en commission et limitant la puissance des éoliennes. De même, le ministre de la Défense, Hervé Morin, a été malmené par l'Assemblée nationale début juin 2009, lorsque le président de la commission des lois, Jean-Luc Warsmann, a émis un avis défavorable sur les nouvelles dispositions régissant le secret défense (il s'opposait à la création de «lieux classifiés»). Mais une intervention personnelle du Premier ministre a permis d'aboutir à une solution de compromis $^{52}$. La nécessité impérieuse de rester au plus près du projet initial pourrait être de nature à rigidifier encore un peu plus la discipline de vote dans les groupes politiques représentés au Parlement. Devant parfois revenir à son texte initial, le Gouvernement sera aussi probablement tenté d'utiliser de manière plus fréquente ses armes constitutionnelles ${ }^{53}$. Et l'on constate déjà que la technique du vote bloqué (art. 44 al. 3 de la Constitution), qui était d'utilisation très exceptionnelle, a été fortement réactivée depuis l'entrée en vigueur de la réforme constitutionnelle en mars $2009^{54}$. Le vote de la loi sur les retraites en octobre 2010 a bien montré la portée limitée des techniques issues de la révision de juillet 2008: alors que les syndicats se réunissaient afin de décider de leurs actions à venir contre la réforme des retraites, le Gouvernement a décidé d'accélérer le mouvement, en combinant les outils constitutionnels mis à sa disposition: au-delà de la mise en œuvre de la procédure accélérée, le ministre du Travail Éric Woerth a demandé devant le Sénat le recours à la procédure dite de vote bloqué, de manière à écourter les débats (il restait alors deux cent cinquante-sept amendements à débattre) ${ }^{55}$.

Réforme indéniablement inaboutie, la révision constitutionnelle du 23 juillet 2008 l'est aussi - et surtout - parce qu'elle ne permet pas de résoudre des problèmes encore plus graves, qui renvoient aux déséquilibres intrinsèques et structurels de la Vépublique.

49. Le président de l'Assemblée nationale, Bernard Accoyer, avait fait part de son souhait d'une procédure «non accélérée» et «sereine» pour l'examen du texte et avait ajouté que le président du Sénat, Gérard Larcher, avec qui il s'en était entretenu, était « sur la même ligne» que lui. De même, le sénateur UMP des Yvelines, Alain Gournac, favorable à la loi sur l'interdiction de la Burqa, s'était dit en revanche «défavorable à la procédure d'urgence» (F. Vignal, «Burqa: le Parlement grince des dents sur la procédure d'urgence», Public Sénat, 23 avril 2010, http://www.publicsenat. fr/lcp/politique/burqa-parlement-grince-des-dents-procedure-d-urgence-12455?quicktabs_1=1\&quicktabs_2=1).

50. Sénat, service des commissions, "Contrôle de l'application des lois pour 2009", 16 décembre 2009 (souligné dans le texte). Le Sénat ajoute même que parmi les 19 autres lois promulguées en 2008-2009, «le nombre de lois votées en procédure d'urgence de droit a été inhabituel: loi de finances initiale, loi de financement de la sécurité sociale, et 4 lois de finances rectificatives en raison de la crise financière, soit 6 lois votées en urgence de droit. Ainsi 13 lois seulement sur 38, soit $34 \%$ du total, ont été examinées selon la procédure de droit commun».

51. Voir J.-C. Colliard, «L'article $16 »$, Les petites affiches, n 97,2008 , p. 54-55.

52. Voir S. Huet, «2009, l'année du nouveau Parlement», Le Figaro, 22 décembre 2009. Voir également "Secret-défense: les députés trouvent un compromis», 9 juin 2009 (http://tempsreel.nouvelobs.com/politique/20090609.OBS9837/les-deputes-parviennent-a-un-compromis-sur-lesecret-defense.html).

53. Voire le chantage: on sait ainsi que Christine Albanel, alors ministre de la Culture, avait menacé de présenter sa démission si le projet de loi "Internet et création» (loi HADOPI) n'était pas adopté au printemps 2009 (l'Assemblée nationale avait alors rejeté la version du texte issue de la commission mixte paritaire) (http://www.lexpress.fr/actualite/politique/albanel-partira-si-la-loi-hadopi-n-est-pas-votee_753344.html).

54. Le comité Balladur était d'ailleurs conscient de ce problème qu'il reconnaissait au moins implicitement. Il indiquait, en effet, que «compte tenu des nombreuses propositions qu'il formule par ailleurs aux fins de renforcer l'autonomie des assemblées, [...] rien ne s'opposait à ce que l'article 44, alinéa 3, de la Constitution fût maintenu en l'état» (Une Ve République plus démocratique, p. 36).

55. Cette décision constituait un véritable désaveu du président UMP du Sénat, Gérard Larcher, qui avait promis que le débat sur la réforme irait «jusqu'au bout» et qui avait avoué être «très réticent» à tout recours à une procédure, comme le vote bloqué, pour l'accélérer. François Fillon lui-même avait affirmé que le Gouvernement souhaitait «un vote à scrutin public d'ici la fin de la semaine», selon des propos rapportés par le ministre du Travail Éric Woerth («Retraites: le Gouvernement choisit de passer en force au Sénat», La Tribune, 21 octobre 2010). 


\section{B. Une réforme ne corrigeant pas les déséquilibres de la Ve République}

Régime parlementaire rationalisé en 1958, la V ${ }^{\mathrm{e}}$ République est devenue, au fil de son histoire, un régime difficilement classable, que certains ont tenté de théoriser, faute de mieux, en régime semi-présidentiel à partir de 1962, avec l'émergence, continue depuis lors, d'un président de la République investi par le suffrage universel direct. En outre, l'apparition dès 1962 de ce que l'on appellera le «fait majoritaire» (à savoir un lien indéfectible entre la majorité de l'Assemblée nationale et le Gouvernement) modifiera les règles du jeu politique, en particulier parce que le texte de 1958 avait été rédigé

[...] dans la perspective d'une absence de majorité forte au Parlement, ce qui fait mieux comprendre les pouvoirs importants donnés au Gouvernement dans la procédure législative lui permettant de faire adopter la loi, y compris contre le Parlement, la procédure de l'article 49, alinéa 3 en étant l'illustration la plus frappante ${ }^{56}$.

Régime atypique inclassable, la $\mathrm{V}^{\mathrm{e}}$ République présente aujourd'hui les apparences d'un régime parlementaire, mais qui s'est fortement présidentialisé, depuis la révision constitutionnelle no 62-1292 du 6 novembre 1962, confirmée et approfondie par la mise en œuvre du quinquennat présidentiel en $2000^{57}$ et par l'«inversion du calendrier électoral» qui, depuis 2002, lie étroitement scrutin présidentiel et élections législatives. C'est dans ce contexte institutionnel, politique et juridique très particulier que s'insère la révision constitutionnelle du 23 juillet 2008. Et, comme l'indique très justement Xavier Vandendriessche, on est en droit de se demander

[...] si cette révision n'a pas tenté de réaliser une équation impossible: atténuer les effets du parlementarisme rationalisé de 1958 sans remettre en cause la prééminence de l'exécutif dans la «détermination» de la conduite de la Nation ${ }^{58}$.

Il convient de ne pas oublier que, depuis 1958, le Gouvernement est chargé de déterminer et de conduire la politique de la Nation (art. 20 de la Constitution). Que l'on doive aujourd'hui substituer le mot "exécutif» au mot «Gouvernement » dans le libellé de cette disposition ne change pas les choses: maître des choix essentiels, le pouvoir exécutif «dispose» du Parlement ${ }^{59}$ et celui-ci n'est chargé que de transcrire en termes législatifs l'action politique de cet exécutif.

Face à de tels déséquilibres structurels, la réforme de 2008 apparaît de portée et d'intensité trop limitées pour modifier les choses en profondeur. Elle échoue notamment à réformer deux dérives de la $\mathrm{V}^{\mathrm{e}}$ République: l'une étant le poids excessif de la législation déléguée, l'autre l'absence de véritables contrepoids internes à l'action de la majorité parlementaire.

Avec l'article 38 de la Constitution, on sait que le Gouvernement peut demander au Parlement, pour une durée limitée, d'adopter par ordonnances des mesures qui ressortent normalement du domaine de loi. Constitutionnalisant et encadrant ainsi les « décrets lois » de la III ${ }^{\mathrm{e}}$ République, le texte de 1958 offre au pouvoir exécutif (le président de la République devant signer les ordonnances) un outil lui permettant de contourner certaines contraintes législatives et d'accélérer la mise en œuvre de ses choix essentiels. L'intérêt de cette procédure a vite été compris par les gouvernements successifs de la $\mathrm{V}^{\mathrm{e}}$ République, qui y ont eu recours de manière massive. Ceci à tel point que dans son Rapport public pour 2006, le Conseil d'État a relevé que les ordonnances sont devenues le principal mode de législation. Les chiffres n'ont pas ralenti depuis, puisque de 2005 à 2010, cent quatre-vingt-huit ordonnances ont été publiées, soit plus que le nombre total d'ordonnances publiées sur vingt ans de 1984 à $2003^{60}$. Sur cette même période, seule la moitié des textes matériellement parlementaires (hors conventions internationales) a suivi la voie parlementaire. Or, la révision de 2008 n'affecte que de manière marginale ces ordonnances. Elle se limite, en effet, à prohiber leur ratification implicite, le deuxième alinéa de l'article 38 précisant désormais qu' «elles ne peuvent être ratifiées que de manière expresse». Cette précision - certes importante - ne résout ni la question des ordonnances "massives ${ }^{61}$ (qui ne permettent pas d'envisager un véritable débat parlementaire lors de la ratification), ni n'oblige l'inscription à l'ordre du jour des projets de loi de ratification (l'article 38 de la Constitution continuant à n'évoquer que le « dépôt » de ces projets). La révision de 2008, contrairement au comité Balladur, n’a pas plus envisagé la question pourtant problématique, de la transposition des directives européennes en droit français. Or, celles-ci sont souvent transposées par la voie

56. «Les noces d'or de la Constitution», entretien avec B. Mathieu et M. Verpeaux, propos recueillis par J. Béguin et H. Béranger, La semaine juridique, éd. $G, \mathrm{n}^{\circ} 40,1^{\text {er }}$ octobre $2008, \mathrm{I}, 189$, p. 38.

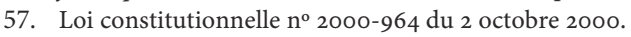

58. X. Vandendriessche, "Une revalorisation parlementaire...», \$2. En 2001, cet auteur faisait d'ailleurs déjà de la modification de l'article 20 de la Constitution «la condition sine qua non pour réaffirmer la nature parlementaire de nos institutions et rendre au Parlement la capacité d'initiative qu'il a globalement perdue en 1958» («Le Parlement entre déclin et modernité», Pouvoirs, nº 99, 2001/4, p. 62, http://www.cairn. info/revue-pouvoirs-2001-4-page-59.htm).

59. Comme le Gouvernement « dispose» constitutionnellement de l'administration et de la force armée, en vertu de l'article 20 alinéa 2 de la Constitution.

60. Voir P. Jan, Les assemblées parlementaires françaises, Paris, La documentation française (Les études), 2010, p. 108.

61. Telles que la loi no $2007-224$ du 21 février 2007 portant dispositions statutaires relatives à l'outre-mer, qui a ratifié vingt-neuf ordonnances, dont vingt-cinq de l'article 38. La loi no 2009-594 pour le développement économique de l'outre-mer du 27 mai 2009, en ratifie dix. Voir P.-Y. Gahdoun, «L'amélioration de la fabrication des lois, entre rénovation et révolution», L'actualité juridique. Droit administratif, 13 octobre 2008 , p. 1892. 
de l'article 38 de la Constitution ${ }^{62}$, ce qui a pour effet de dessaisir le Parlement de questions importantes, qui, pour certaines d'entre elles en tout cas, renvoient à des enjeux politiques essentiels ${ }^{63}$.

Si la révision de 2008 échoue en grande partie dans sa volonté de restaurer un relatif équilibre entre les pouvoirs, le même constat peut être effectué concernant le rééquilibrage interne du Parlement. Si le Parlement apparaît trop faible dans sa fonction législative, c'est notamment parce que la force de la majorité parlementaire est excessive et cantonne l'opposition à un rôle de faire-valoir sans réelle portée. On sait qu'en France, la «culture» de l'opposition est particulièrement réduite ${ }^{64}$, comme a pu le démontrer la position de principe adoptée par le Conseil constitutionnel en 2006: refusant la consécration de la notion d' "opposition», il indique qu'une telle reconnaissance serait de nature à rompre le principe d'égalité entre les partis politiques (le droit français ne reconnaissant que les partis, il n'était pas possible d'accorder des droits spécifiques à cette opposition ${ }^{65}$ ). Les choses ont toutefois quelque peu évolué depuis et l'on sait notamment que l'article 39 alinéa 3 du Règlement de l'Assemblée nationale prévoit désormais que ne peut être élu à la présidence de la commission des finances qu'un député appartenant à un groupe d'opposition. À compter de la prochaine législature, il en ira de même pour la présidence de la commission spéciale chargée de vérifier et d'apurer les comptes de l'Assemblée (art. 16 RAN) ${ }^{66}$.

Certes, la loi constitutionnelle du 23 juillet 2008 , suivant en cela le chemin emprunté par le comité Balladur ${ }^{67}$, n'est pas restée totalement muette sur cette question de l'opposition. On sait notamment que l'article 51-1 de la Constitution indique désormais que

[1]e règlement de chaque assemblée détermine les droits des groupes parlementaires constitués en son sein. Il reconnaît des droits spécifiques aux groupes d'opposition de l'assemblée intéressée ainsi qu'aux groupes minoritaires.

Ceci s'avère en pratique assez limité car cette nouvelle disposition ne précise en rien quels sont les nouveaux droits concrètement reconnus à ces groupes d'opposition, ou minoritaires, en dehors de la fixation de l'ordre du jour... une fois par mois (art. 48 al. 5 de la Constitution) ${ }^{68}$. Ces quelques dispositions sont, en outre, très en retrait par rapport à ce qu'avait pu envisager le comité Balladur ${ }^{69}$ et même par rapport à la version initiale du projet de loi

62. Ce qui avait assez fortement ébranlé la cohésion de la majorité à la fin de l'année 2000 avec l'utilisation par le Gouvernement de la technique des ordonnances pour transposer en bloc plusieurs directives. Pour le Premier ministre, ce choix était commandé par l'urgence, car «au moment où nous assumions la présidence française (de l’Union européenne), la France se trouvait avec 136 directives en retard de transposition » (J.-M. Bezat, "La transposition de directives ébranle à nouveau la cohésion de la majorité», Le Monde, 6 et 7 décembre 2000).

63. À ce titre, le comité Balladur dans sa proposition $n^{\circ} 52$ proposait que soit utilisée, en matière de ratification des directives européennes, la procédure simplifiée d'examen en commission, sauf difficulté particulière signalée par le Comité des affaires européennes. Ceci aurait permis "de réserver à la discussion publique les questions les plus importantes et aurait revalorisé le rôle du Parlement " (Une Ve République plus démocratique, p. 62).

64. À la différence de certains États voisins, comme l'a relevé le comité Balladur, «le statut de l'opposition au Royaume-Uni [a] bien la valeur d'exemple qu'on lui prête: depuis 1826 , le parti possédant le plus grand nombre de députés après le parti au pouvoir constitue l'opposition et bénéficie, en cette qualité, de prérogatives propres; le chef de l'opposition y dispose, depuis 1937, d'un statut officiel; à la Chambre des Communes, l'opposition préside un tiers des commissions dont celle chargée du contrôle du budget et des comptes; une partie de l'ordre du jour est laissée à sa disposition. En République Fédérale d'Allemagne, les présidences des 22 commissions du Bundestag et celles des commissions d'enquête sont réparties à la proportionnelle des groupes, la commission des finances est présidée par un membre du principal parti d'opposition» (Une Ve République plus démocratique, p. 66).

65. CC, déc. $\mathrm{n}^{\circ}$ 2006-537 DC du 22 juin 2006. Voir P.-Y. Gahdoun, «L'amélioration de la fabrication des lois... », p. 1877.

66. De même, le bureau des commissions législatives doit s'efforcer de reproduire la configuration politique de l'Assemblée nationale et d'assurer la représentation de toutes ses composantes (art. 39 RAN). Une règle identique est prévue pour la commission spéciale chargée de vérifier et d'apurer les comptes de l'Assemblée nationale (art. 16 al. 2 RAN), pour les commissions d'enquête (art. 143 al. 2 RAN) et pour les missions d'information créées par la Conférence des présidents sur proposition du président de l'Assemblée (art. 145 al. 4 RAN). Voir également l'art. 6 bis al. 4 du Règlement du Sénat relatif aux fonctions de président et de rapporteur d'une commission d'enquête ou d'une mission d'information.

67. La lettre de mission de ce comité démontre l'importance attachée à la question de l'opposition: «une démocratie exemplaire, c'est aussi une démocratie qui veille à ce que l'opposition ait les moyens d'exercer son rôle, qu'il s'agisse de moyens politiques, juridiques, ou financiers. C'est pourquoi je souhaite que le comité me propose un statut de l'opposition, définissant cette dernière et lui reconnaissant un certain nombre de droits» (Lettre de mission du président de la République du 18 juillet 2007, in Une Ve République plus démocratique, annexe 2, p. 209).

68. La description des premières mises en œuvre de cette possibilité laisse toutefois perplexe: «grâce à la révision constitutionnelle de juillet 2008 , l'opposition a "multiplié par trois" le temps dont elle dispose pour inscrire à l'ordre du jour des propositions de son choix, proclament fièrement les responsables de la majorité. La réalité est moins exaltante. À ces textes soumis à la discussion par l'opposition lors de ces séances traditionnellement organisées le jeudi, la majorité oppose la résistance par le vide. Premier temps, en conférence des présidents, le "patron" du groupe UMP, Jean-François Copé, demande un scrutin solennel sur chacun des textes. Le vote sera donc différé au mardi, après les questions au Gouvernement. Deuxième temps, le Gouvernement demande, en application de l'article 44-3 de la Constitution, la "réserve" des votes sur les articles et les amendements en discussion. Dès lors, aucun vote ne sera appelé. Troisième temps, la majorité, ne courant aucun risque d'être mise en minorité, laisse l'opposition débattre seule» (P. Roger, «Le Parlement apprend, tant bien que mal, à faire usage de ses nouveaux droits », Le Monde, 24 décembre 2009).

69. Il proposait la mise en place de "garanties nouvelles aux groupes qui ne soutiennent pas le Gouvernement», ce qui renvoyait tout à la fois au partage de l'ordre du jour, à la répartition des temps de parole, à la création des commissions d'enquête et au choix de leurs rapporteurs, à la présidence des commissions permanentes, au mode de désignation des organismes extérieurs chargés de représenter le Parlement (propositions $\mathrm{n}^{\circ} 57$ et 58). Il préconisait également la reformulation de l'article 4 de la Constitution afin d'y écrire que la loi détermine les conditions dans lesquelles sont garantis les droits des partis et groupements qui ont ou n’ont pas déclaré soutenir le Gouvernement (proposition n 60 ), ainsi que la rédaction d'une «Charte des droits de l'opposition» de caractère non normatif, mais de nature à garantir «les bonnes pratiques d'une démocratie parlementaire plus respectueuse des opinions et des personnes» (Une Ve République plus démocratique, p. 65 sq.). 
constitutionnelle ${ }^{70}$. Les modifications des règlements des deux assemblées démontrent que les droits et garanties concrètes reconnus aux groupes minoritaires et d'opposition ont été extrêmement limités. Les nouveaux modes d'organisation du temps de parole pourraient notamment être de nature à fortement limiter les modalités d'action de l'opposition parlementaire ${ }^{71}$.

Que penser au final de la revalorisation du Parlement opérée par la révision constitutionnelle du 23 juillet 2008 ? Il ne s'agit, à l'évidence, ni d'un catalogue de «mesurettes", ni du «grand soir» du Parlement français. Et le manque de recul face à l'application de réformes entrées en vigueur il y a un peu plus de dix-huit mois est un exercice difficile. On peut surtout penser que la portée des différentes réformes risque de se diluer progressivement, en raison de pratiques gouvernementales contraires à l'esprit de la réforme. La réussite de la valorisation du Parlement (mais également de sa mission de contrôle) réside, en effet, dans un changement de mentalité des acteurs politiques ${ }^{72}$ et l'on peut douter que le pouvoir exécutif - en particulier à l'approche d'échéances politiques majeures - accepte spontanément de desserrer l'étreinte qu'il maintient sur le Parlement depuis maintenant près de quarante années. Nous n'avons donc d'autre choix que de faire nôtres les conclusions du comité Vedel qui, en 1993, soulignait avec sagesse (ou résignation?) que «la règle de droit est le seul opérateur immédiatement disponible (à défaut de l'évolution des croyances et des mœurs) pour obtenir un résultat institutionnel. Convenablement utilisée, sans naïveté mais sans excès de scepticisme, elle peut changer quelque chose en bien ${ }^{73}$.

Caen, novembre 2010

70. Qui prévoyait initialement dans son article $1^{\mathrm{er}}$ (modifiant l'article 4 de la Constitution) un principe général de reconnaissance de l'opposition, définie comme l'ensemble des partis et groupements politiques qui n'ont pas déclaré soutenir le Gouvernement. L'article 24 précisait que le règlement de chaque assemblée déterminerait les droits spécifiques des groupes parlementaires selon qu'ils ont ou non déclaré soutenir le Gouvernement. L'Assemblée nationale, en première lecture, a estimé que l'article $1^{\text {er }}$ n'avait pas qu'une utilité marginale par rapport à l'article 24. Elle a donc préféré préciser, dans l'article 4 de la Constitution, que la loi doit garantir la participation des partis et groupements politiques à la vie démocratique de la Nation. Le Sénat l'a suivie en précisant que cette reconnaissance devait se faire "dans le respect du pluralisme» (J.-L. Warsmann, Rapport no $1009 . .$.$) .$

71. Ainsi, à l'Assemblée nationale, le temps de parole alloué à un député passe de cinq à deux minutes pour la discussion d'un article, comme pour la discussion d'un amendement (art. 95 al. 2 et 10o RAN). Sur le contenu de ces droits, voir notamment H. Jozefowicz, «La réforme des règlements des assemblées parlementaires: entre impératifs constitutionnels, amélioration du débat et ouverture au parlementarisme», Revue française de droit constitutionnel, $\mathrm{n}^{\circ}$ 82, 2010, p. 329 sq.; A. Vidal-Naquet, "L'institutionnalisation de l'opposition. Quel statut pour quelle opposition?», Revue française de droit constitutionnel, $\mathrm{n}^{\circ} 77,2009$, p. 153 sq.

72. Voir, notamment, X. Vandendriessche, «Une revalorisation parlementaire...», $\$ 60$.

73. Rapport remis au Président de la République le 15 février 1993..., p. 2538. 